\title{
Proton-Air Cross Section and Extensive Air Showers
}

\author{
Ralf Ulrich $^{\mathrm{a}}$, Ralph Engel ${ }^{\mathrm{a}}$, Steffen Müller ${ }^{\mathrm{a}}$, Fabian Schüssler ${ }^{\mathrm{a}}$ and Michael Unger ${ }^{\mathrm{a}}$ \\ ${ }^{a}$ Karlsruhe Institute of Technology (KIT) \\ Institut für Kernphysik, P.O. Box 3640, 76021 Karlsruhe, Germany \\ (KIT is the cooperation of University Karlsruhe and Forschungszentrum Karlsruhe)
}

\begin{abstract}
Hadronic cross sections at ultra-high energy have a significant impact on the development of extensive air shower cascades. Therefore the interpretation of air shower data depends critically on hadronic interaction models that extrapolate the cross section from accelerator measurements to the highest cosmic ray energies. We discuss how extreme scenarios of cross section extrapolations can affect the interpretation of air shower data. We find that the theoretical uncertainty of the extrapolated proton-air cross section at ultra-high energies is much larger than suggested by the existing spread of available Monte Carlo model predictions. The impact on the depth of the shower maximum is demonstrated.
\end{abstract}

\section{Introduction}

The inherent uncertainties of the modeling of hadronic interactions at ultra-high energies are still preventing an unambiguous analysis of existing cosmic ray data. They introduce large and difficult to quantify systematic uncertainties on many cosmic ray analyses. This is a well known problem for the primary energy spectrum as derived from Monte Carlo calibrated surface detector data [1-6], as well as for the determination of the primary mass composition of high energy cosmic rays [5,7-11]. The model-dependence of the interpretation of air shower data is mainly related to the differing predictions of the number of muons in air showers and the depth of the shower maximum [12].

It is unclear whether the existing differences in model predictions can be used to estimate the current theoretical systematic uncertainties caused by inaccurate modeling of hadronic interactions in air showers. It is possible that existing model-differences are

(A) overestimating the current systematic uncertainties. Better understanding of hadronic particle production and new data from accelerators allow us to update interaction models to obtain a more realistic description of particle production. Not all models are updated regularly and the qual- ity of data description differs between the models.

(B) underestimating the current systematic uncertainties, because the existing models are by far not sampling the full phase space of possible interaction scenarios and parameters. Moreover, new physics at higher energies may be unknown and is thus even missing in current approaches.

Of the frequently used models, there seem to be more sophisticated and possibly better models, e.g. QGSJET II $[13,14]$ and Epos [15], compared to others that are older and more simplistic or specialized, e.g. QGSJET $01[16,17]$ and Sibyll $2.1[18,19]$. Furthermore it must be kept in mind that many other models are considered as outdated or just not fashionable like NEXus [20,21], HDMP [22], DPMJET [23], DTUJET [24], VENUS [25] etc. . This classification is very subjective; Different communities make differing choices.

The current situation is characterized by the fact that none of the models is able to consistently describe cosmic ray data, and the predictions by SibYLL 2.1 and QGSJet 01 are not objectively worse than the ones by QGSJetII and Epos. Over the years model predictions and extrapolations have become more alike even though there is no theory for calculating e.g. such cross sec- 


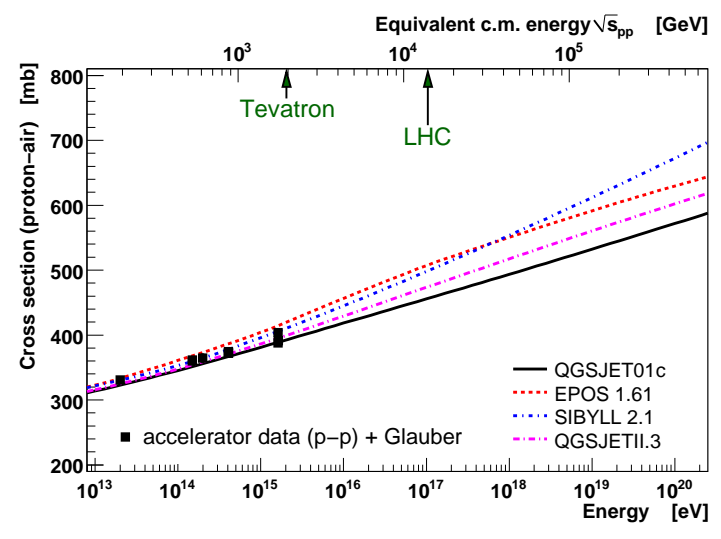

Figure 1. Extrapolation of the proton-air production cross section from accelerator measurements (see [26] for references) to cosmic ray energies. The accelerator measurements ( $\mathrm{p}-\mathrm{p}$ and $\mathrm{p}-\overline{\mathrm{p}})$ are converted to $\mathrm{p}$-air within the framework of the respective interaction model.

tions from first principles. This is demonstrated in Fig. 1 by comparing the predictions on the total proton-air cross section for secondary particle production. On the other hand, recent activity in model development within the EPos event generator clearly demonstrated that there exists a relatively large freedom to tune model predictions at ultra-high energies [27]. All this is indicating that the current situation corresponds to option (B).

The aim of this work is to start from existing accelerator measurements and investigate the importance of different cross section extrapolations. The theoretical formalism to calculate cross sections of hadronic projectiles with air is described and the available accelerator data are discussed. Based on these considerations different extrapolations of the existing data to ultra-high energy are considered and the resulting impact on the development and, thus, interpretation of cosmic ray induced air showers are discussed.

\section{Glauber Formalism}

For the development of extensive air showers the cross sections of hadronic projectiles with air at center of mass energies from $\mathrm{GeV}$ to several hundreds of $\mathrm{TeV}$ are relevant. These cross sections have to be calculated from models based on data for nucleon-nucleon and meson-nucleon scattering that has been studied at accelerators.

It was realized by Glauber $[28,29]$ that the scattering amplitude in the impact parameter space can be treated in analogy to diffraction in optics by using the phase shift relation

$$
a(s, b)=1-e^{\mathrm{i} \chi(s, b)},
$$

with the Eikona 1 function $\chi$. Within this picture of neglecting the recoil due to the individual nucleon-nucleon interactions, multiple scattering can be written as

$$
\begin{aligned}
a_{h A}(s, b) & =1-e^{\mathrm{i} \chi_{\operatorname{mult}}(s, b)} \\
& =1-\prod_{j}\left(1-a_{j}\left(s, b_{j}\right)\right),
\end{aligned}
$$

whereas the overall phase shift $\chi_{\text {mult }}$ corresponds to the sum of the individual phase shifts $\chi_{j}$ for each scattering process $j$.

Figure 2 illustrates the interaction of a projectile particle with a nucleus. According to Eq. (2) the projectile scatters separately with each of the nucleons $j$ of the nucleus. The resulting total and elastic cross section are given by

$$
\begin{aligned}
\sigma_{\text {tot }}^{\mathrm{hA}} & =2 \Re e \int \Gamma_{h A}(\vec{b}) \mathrm{d}^{2} b, \\
\sigma_{\text {ela }}^{\mathrm{hA}} & =\int\left|\Gamma_{h A}(\vec{b})\right|^{2} \mathrm{~d}^{2} b,
\end{aligned}
$$

with

$$
\Gamma_{\mathrm{hA}}(\vec{b})=1-\prod_{j=1}^{A}\left[1-\int a_{j}\left(\vec{b}-\vec{b}_{j}\right) \rho_{j}\left(\vec{r}_{j}\right) \mathrm{d}^{3} r_{j}\right] .
$$

For clarity of the expressions we have applied the simplification of non-correlated nucleons

$$
\psi^{\star}\left(\vec{r}_{1} \ldots \vec{r}_{A}\right) \psi\left(\vec{r}_{1} \ldots \vec{r}_{A}\right)=\prod_{j=1}^{A} \rho_{j}\left(\vec{r}_{j}\right),
$$

\footnotetext{
$\overline{{ }^{1} \text { From the Greek word }} \epsilon \iota \kappa \omega \nu$ with the meaning of image.
} 


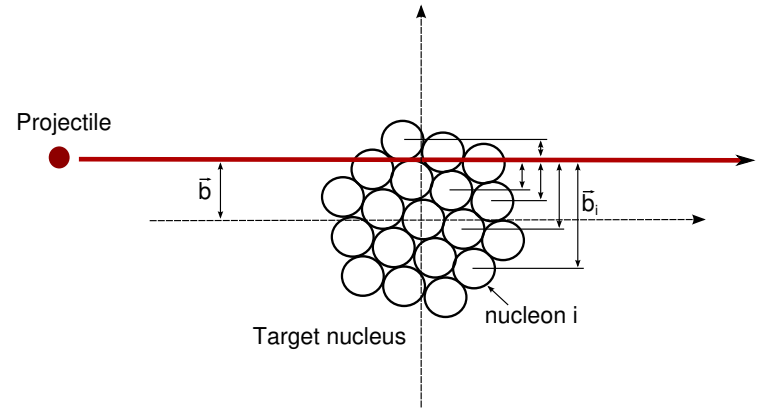

Figure 2. A projectile particle hitting a target nucleus with an impact parameter $\vec{b}$. The relative impact parameter $\vec{b}_{j}$ for each of the nucleons governs the scattering of the projectile from the individual nucleon $i$.

where $\psi$ denotes the wave function of the nucleus with $\vec{r}_{i}$ being the coordinates of the individual nucleons $j=1 \ldots A$. The impact parameters of the nucleons are denoted by $\vec{b}_{j}$ and that of the cosmic ray hadron by $\vec{b}$. The single nucleon density is normalized to $\int \rho_{j}\left(\vec{r}_{j}\right) \mathrm{d}^{3} r_{j}=1$. The sum of the cross sections for elastic and quasi-elastic scattering is given by

$$
\begin{array}{r}
\sigma_{\text {ela }}^{\mathrm{hA}}+\sigma_{\mathrm{qel}}^{\mathrm{hA}}=\int \mathrm{d}^{2} b\left|1-\prod_{j=1}^{A}\left[1-a_{j}\left(\vec{b}-\vec{s}_{j}\right)\right]\right|^{2} \\
\times\left(\prod_{j=1}^{A} \rho_{j}\left(\vec{r}_{j}\right) \mathrm{d}^{3} r_{j}\right) .
\end{array}
$$

Knowing the nucleon-nucleon impact parameter amplitudes $a_{j}\left(s, \vec{b}-\vec{b}_{j}\right)$ allows one to calculate the multiple scattering amplitude and hence the total, elastic and quasi-elastic cross sections.

Based on the optical theorem the nucleonnucleon scattering amplitude is typically parametrized as

$$
a_{j}\left(s, b_{j}\right)=\frac{(1+\rho(s)) \sigma_{\text {tot }}^{\mathrm{pp}}(s)}{4 \pi B_{\text {ela }}(s)} \mathrm{e}^{-{\overrightarrow{b_{j}}}^{2} /\left(2 B_{\text {ela }}(s)\right)},
$$

where $\rho$ is the ratio of the real to imaginary part of the forward scattering amplitude [30], and
$B_{\text {ela }}$ is the elastic slope parameter defined by $\mathrm{d} \sigma_{\text {ela }} /\left.\mathrm{d} t\right|_{t=0} \propto \mathrm{e}^{-B_{\text {ela }}|t|}$.

It is the combination of expressions (3) and (7) that finally makes the connection between nucleon-nucleon and nucleon-nucleus scattering. With these formulations it is possible to calculate the total, elastic and quasi-elastic cross section for nucleon-nucleus scattering just from the parameters $B_{\text {ela }}, \rho, \sigma_{\text {tot }}^{\text {pp }}$ and the possible configurations of the nucleus.

It can be shown that the Glauber approximation follows from Gribov's Regge calculus if inelastic intermediate states are neglected [31]. The inclusion of intermediate states leads to inelastic screening corrections. SIBYLL 2.1 is a model that is based on the classical Glauber approximation. Inelastic screening corrections are implemented in QGSJET II within Gribov's Reggeon calculus. The cross section calculation in EPOS is accounting for high parton density effects with a parametrization of the expected screening effects. There are other models of amplitudes and corresponding schemes of describing multiple scattering that are not available in any event generator, see for example [32].

\section{Accelerator Data and their Extrapola- tion to Cosmic Ray Energies}

Up to $\sqrt{s} \sim 2 \times 10^{3} \mathrm{GeV}$ measurements of $\rho$, $B_{\text {ela }}$ and $\sigma_{\text {tot }}^{\text {pp }}$ are available from accelerator experiments (c.f. [26] and references therein). The experimental precision is best at lower energies. The extrapolation of these data to higher energies, however, is model-dependent and subject to uncertainties (e.g. $[34,36,37])$. For the application in cosmic ray physics at least two orders of magnitude in energy in the center of mass system have to be extrapolated. In Figure [3] the resulting magnitude of uncertainty at cosmic ray energies is demonstrated. While all extrapolations of $\rho$ and $B_{\text {ela }}$ (c.f. Fig. 3. (b) $+(\mathrm{c})$ ) are just phenomenological parametrizations and thus not based on underlying physics. The extrapolation of $\sigma_{\text {tot }}^{\text {pp }}$ (see Fig. 3. (a)) is taken from published work; The conventional model is the soft pomeron parametrization by Donnachie and Landshoff [33], while the lower extreme is a model 


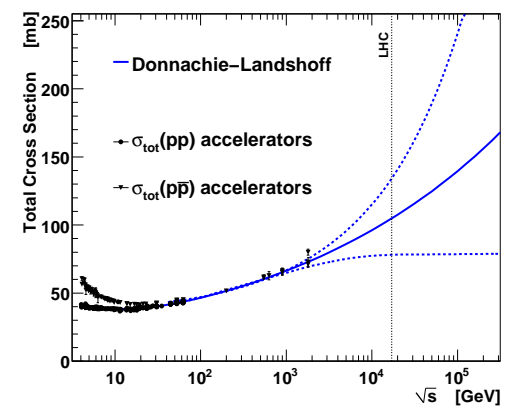

(a) $\sigma_{\text {tot }}^{\text {pp }}$

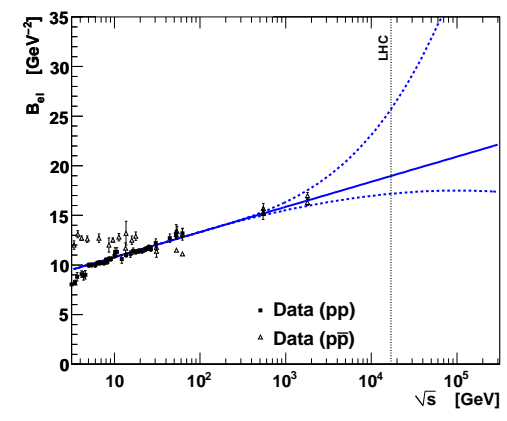

(b) $B_{\text {ela }}$

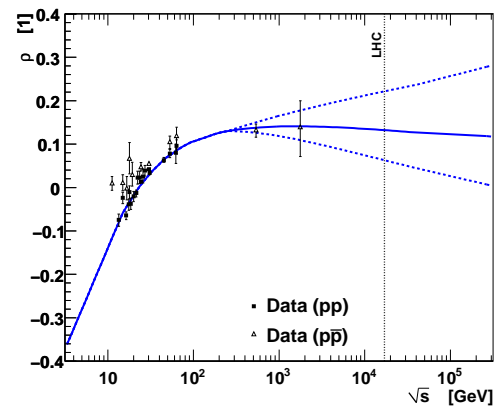

(c) $\rho$

Figure 3. Compilation of accelerator data of $\sigma_{\mathrm{tot}}^{\mathrm{pp}}, B_{\text {ela }}$ and $\rho$ [26]. The central line denotes the conventional extrapolation of these data to high energy. The upper and lower lines indicate a set of possible extreme extrapolations. In the left plot the conventional model is the soft pomeron parametrization by Donnachie and Landshoff [33], while the lower extreme is by Pancheri et al. [34] and the upper extreme is the two-pomeron model of Landshoff $[35,36]$.

by Pancheri et al. [34] and the upper extreme the two-pomeron model by Landshoff $[35,36]$.

\section{Resulting Proton-Air Cross Section}

Combining the extrapolations given in Fig. 3 with Glauber theory it is possible to calculate the proton-air cross section, which is important for air shower development. Typically only the production cross section

$$
\sigma_{\text {prod }}=\sigma_{\text {tot }}-\sigma_{\text {ela }}-\sigma_{\text {qel }}
$$

is quoted in the context of extensive air showers $[38,39]$, since interactions with no new particle production are not relevant to the development of air showers.

The results are shown in Fig. 4. Already at $10^{18} \mathrm{eV}$ the uncertainty band is significantly larger than the range covered by all available interaction models. Around $10^{19} \mathrm{eV}$ the relative uncertainty reaches up to $50 \%$.

\section{Impact on the Interpretation of Cosmic Ray Data}

The choice of the extrapolation of the protonair cross section (Fig. 4) has a strong impact on the predicted speed of shower development and thus on the depth of the shower maximum [43].
The typical interpretation of $\left\langle X_{\max }\right\rangle$ data in terms of a mixed mass composition at high energy (Fig. 5] left) has to be revised if a different cross section extrapolation is used. As shown in Fig. [5 (right), the data could also be explained with a cross section that is increased by $f_{19}=40-60 \%$ at $10^{19} \mathrm{eV}$ in combination with very light cosmic ray primaries. Similar results have been obtained independently in Ref. [44].

In our calculations all hadronic interaction cross sections are increased by a factor that depends logarithmically on energy

$$
f(E)=1+\left(f_{19}-1\right) \frac{\ln \left(E / 10^{15} \mathrm{eV}\right)}{\ln \left(10^{19} \mathrm{eV} / 10^{15} \mathrm{eV}\right)},
$$

for $E>10^{15} \mathrm{eV}$ and $f(E)=1$ otherwise.

\section{Summary}

It is argued that the uncertainties of the extrapolation of hadronic cross sections to cosmic ray energies might be underestimated if only commonly used models are considered. The true uncertainty could be much larger than the one suggested by the spread of the current predictions of hadronic interaction models.

Since longitudinal air shower development depends sensitively on hadronic cross sections, predictions for standard observables like the depth 


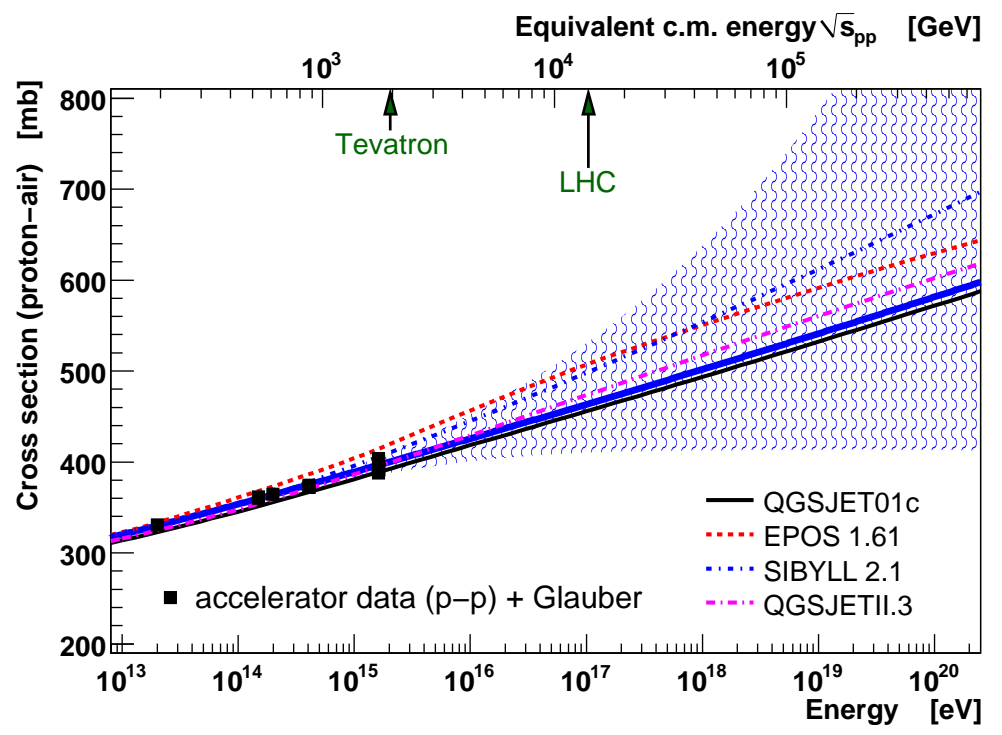

Figure 4. Uncertainty of the extrapolation of the proton-air cross section, $\sigma_{\text {prod }}^{\mathrm{p}-\text { air }}$, from accelerator to cosmic ray energies.
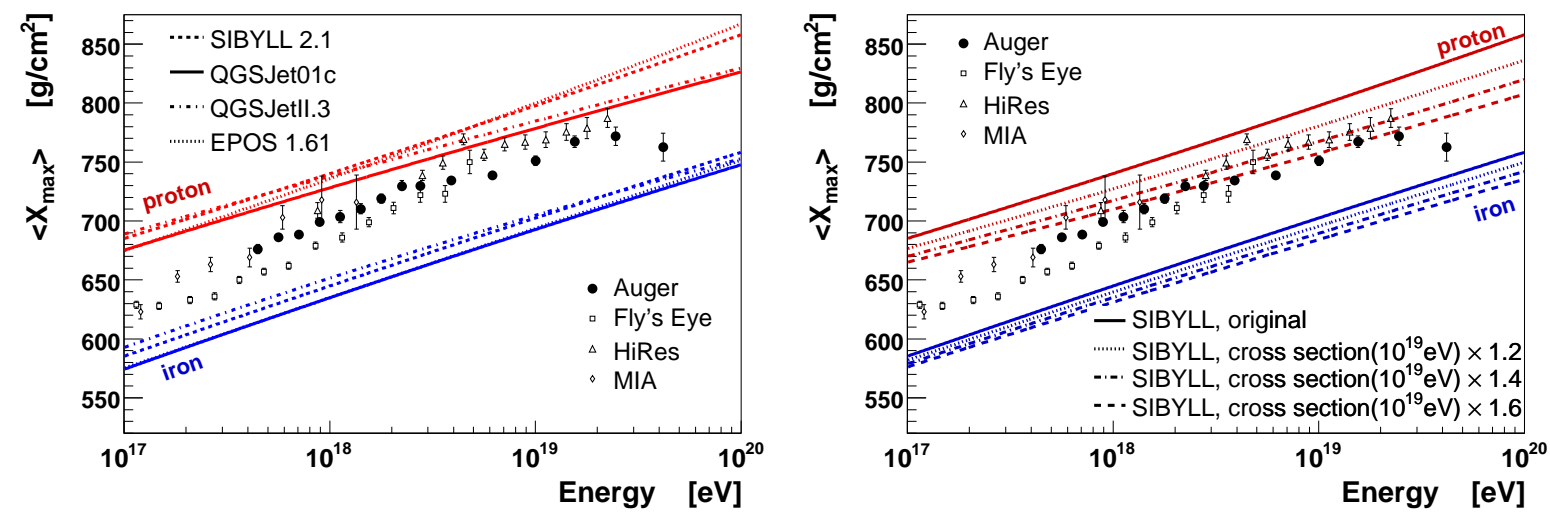

Figure 5. Left panel: Measurements of the mean depth of the shower maximum [10,11,40,41] compared to air shower simulations for different primary particles and interaction models. Right panel: Same data compared to air shower simulations using SIBYLL and a modified cross section extrapolation [42, 43].

of the shower maximum, $X_{\max }$, are strongly affected by these uncertainties. It is shown that the measured mean depth of the shower maximum at ultra-high energies could be explained by a very light cosmic ray mass composition in combination with a modification of the extrapolation of the hadronic cross section to ultra-high energy.

\section{REFERENCES}

1. M. Nagano et al., J. Phys. G18 (1992) 423442.

2. M. Takeda et al. (AGASA Collab.), Phys. Rev. Lett. 81 (1998) 1163-1166 and astroph/9807193. 
3. M. Ave, J. Knapp, J. Lloyd-Evans, M. Marchesini, and A. A. Watson, Astropart. Phys. 19 (2003) 47-60 and astro-ph/0112253.

4. M. Aglietta et al. (EAS-TOP Collab.), Astropart. Phys. 21 (2004) 583-596.

5. T. Antoni et al. (KASCADE Collab.), Astropart. Phys. 24 (2005) 1-25 and astro$\mathrm{ph} / 0505413$.

6. M. Amenomori et al. (Tibet AS $\gamma$ Collab.), Astrophys. J. 678 (2008) 1165-1179 and arXiv:0801.1803 [hep-ex].

7. B. R. Dawson, R. Meyhandan, and K. M. Simpson, Astropart. Phys. 9 (1998) 331-338 and astro-ph/9801260.

8. M. Ave et al., Astropart. Phys. 19 (2003) 6175 and astro-ph/0203150.

9. M. T. Dova, M. E. Mancenido, A. G. Mariazzi, T. P. McCauley, and A. A. Watson, Astropart. Phys. 21 (2004) 597-607 and astro$\mathrm{ph} / 0312463$.

10. R. U. Abbasi et al. (HiRes Collab.), Astrophys. J. 622 (2005) 910-926 and astro$\mathrm{ph} / 0407622$.

11. M. Unger (Pierre Auger Collab.), Proc of 30th Int. Cosmic Ray Conf., Merida 4 (2007) 373 and arXiv:0706.1495 [astro-ph].

12. D. Heck, Talk given at CORSIKA School 2008, Freudenstadt, Germany, 25 - 30 November 2008, available at http://wwwik.fzk.de/corsika/corsika-school2008/, 2008.

13. S. Ostapchenko, Nucl. Phys. Proc. Suppl. 151 (2006) 143-146 and hep-ph/0412332.

14. S. Ostapchenko, Phys. Lett. B636 (2006) 4045 and hep-ph/0602139.

15. K. Werner and T. Pierog, AIP Conf. Proc. 928 (2007) 111-117 and arXiv:0707.3330 [astro-ph].

16. N. N. Kalmykov and S. S. Ostapchenko, Phys. Atom. Nucl. 56 (1993) 346-353.

17. N. N. Kalmykov and S. S. Ostapchenko, Sov. J. Nucl. Phys. 50 (1989) 315-318.

18. R. Engel, T. K. Gaisser, T. Stanev, and P. Lipari, Proc. of 26th Int. Cosmic Ray Conf., Salt Lake City 1 (1999) 415.

19. R. S. Fletcher, T. K. Gaisser, P. Lipari, and T. Stanev, Phys. Rev. D50 (1994) 5710-5731.

20. H. J. Drescher, M. Hladik, S. Ostapchenko, T. Pierog, and K. Werner, Phys. Rept. 350
(2001) 93-289 and hep-ph/0007198.

21. M. Hladik, H. J. Drescher, S. Ostapchenko, T. Pierog, and K. Werner, Phys. Rev. Lett. 86 (2001) 3506-3509 and hep-ph/0102194.

22. J. N. Capdevielle, J. Phys. G15 (1989) 909924.

23. J. Ranft, Phys. Rev. D51 (1995) 64-84.

24. F. W. Bopp, D. Pertermann, R. Engel, and J. Ranft, Phys. Rev. D49 (1994) 3236-3247.

25. K. Werner, Phys. Rep. 232 (1993) 87.

26. R. Engel, Nucl. Phys. Proc. Suppl. 82 (2000) 221-231.

27. T. Pierog and K. Werner, Proc. of 30th Int. Cosmic Ray Conf., Merida 4 (2007) 629.

28. R. J. Glauber, Phys. Rev. 100 (1955) 242-248.

29. R. J. Glauber and G. Matthiae, Nucl. Phys. B21 (1970) 135-157.

30. M. M. Block and R. N. Cahn, Rev. Mod. Phys. 57 (1985) 563.

31. J. H. Weis, Acta Phys. Polon. B7 (1976) 851.

32. S. M. Troshin and N. E. Tyurin, Phys. Lett. B316 (1993) 175.

33. A. Donnachie and P. V. Landshoff, Phys. Lett. B296 (1992) 227-232 and hep-ph/9209205.

34. G. Pancheri, R. M. Godbole, A. Grau, and Y. N. Srivastava, Acta Phys. Polon. B38 (2007) 2979-2988 and hep-ph/0703174.

35. P. V. Landshoff, arXiv:0709.0395 [hep-ph].

36. P. V. Landshoff, arXiv:0903.1523 [hep-ph].

37. M. M. Block, Phys. Rept. 436 (2006) 71-215 and hep-ph/0606215.

38. N. N. Nikolaev, Phys. Rev. D48 (1993) $1904-$ 1906 and hep-ph/9304283.

39. R. Engel, T. K. Gaisser, P. Lipari, and T. Stanev, Phys. Rev. D58 (1998) 014019 and hep-ph/9802384.

40. D. J. Bird et al. (Fly's Eye Collab.), Phys. Rev. Lett. 71 (1993) 3401-3404.

41. T. Abu-Zayyad et al. (HiRes-MIA Collab.), Astrophys. J. 557 (2001) 686-699 and astro$\mathrm{ph} / 0010652$.

42. R. Ulrich, J. Blümer, R. Engel, F. Schüssler, and M. Unger, arXiv:0709.1392 [astro-ph].

43. R. Ulrich et al., in preparation (2009) .

44. T. Wibig, arXiv:0810.5281 [hep-ph]. 